\title{
Negative selection of Plasmodium falciparum reveals targeted gene deletion by double crossover recombination
}

\author{
Manoj T. Duraisingh, Tony Triglia, Alan F. Cowman* \\ The Walter and Eliza Hall Institute of Medical Research, P.O. Royal Melbourne Hospital, Melbourne, Vic. 3050, Australia
}

Received 2 November 2001; accepted 8 November 2001

\begin{abstract}
The genome sequence of Plasmodium falciparum, the causative agent of the most severe form of malaria in humans, rapidly approaches completion, but our ability to genetically manipulate this organism remains limited. Chromosomal integration has only been achieved following the prolonged maintenance of circularised episomal plasmids which selects for single crossover recombinants. It has not been possible to construct genetic deletions via double crossover recombination, presumably due to the low frequency of this event. We have used the Herpes simplex virus thymidine kinase gene and the Escherichia coli cytosine deaminase gene for negative selection of $P$. falciparum. Parasites were transformed with plasmids expressing the thymidine kinase and cytosine deaminase genes by positive selection for the human dihydrofolate reductase gene. Parasites expressing thymidine kinase are susceptible to the pro-drug ganciclovir while those expressing cytosine deaminase are sensitive to 5-fluorocytosine. Parental parasites were inherently resistant to these drugs. A significant 'bystander effect' was evident in cultures with either ganciclovir or 5-fluorocytosine. Positive and negative selection of the thymidine kinase transformants with both ganciclovir and WR99210 resulted in the selection of parasites containing a genetic deletion of the Pfrh3 gene, the first targeted double crossover deletions in $P$. falciparum. The use of negative selection for gene disruptions via double crossover recombination will dramatically improve our ability to analyse protein function and opens the possibility of using this strategy for a variety of gene deletion and modification experiments in the analysis of this important infectious agent. (C) 2002 Australian Society for Parasitology Inc. Published by Elsevier Science Ltd. All rights reserved.
\end{abstract}

Keywords: Malaria; Recombination; Transfection; Plasmodium falciparum; thymidine kinase

\section{Introduction}

The severest form of malaria in humans is caused by infection with the protozoan parasite Plasmodium falciparum. The global burden of disease is worsening in the face of increasing drug-resistance and currently there is no vaccine. Recently much effort has gone into the sequencing of the 14 chromosomes of the P. falciparum genome and to date both chromosomes 2 and 3 have been fully sequenced (Gardner et al., 1998; Bowman et al., 1999). Exploiting this valuable resource will further our understanding of the roles of the proteins encoded by these genes in important processes such as parasite invasion of host cells, molecular pathogenesis and drug resistance, with the aim of identifying new targets for the development of vaccines and novel drug targets.

Recently $P$. falciparum has become more amenable to recombinant DNA technology (Crabb and Cowman, 1996;

\footnotetext{
* Corresponding author. Tel.: +61-3-9345-2555; fax: +61-3-9347-0852.

E-mail address: cowman@wehi.edu.au (A.F. Cowman).
}

$\mathrm{Wu}$ et al., 1996) and the functional analysis of parasite genes has become possible by gene disruption (Crabb et al., 1997a), allelic exchange (Triglia et al., 1998) and transgene expression (Crabb et al., 1997b; Waller et al., 2000). These have been established using positive selection for the presence of circular plasmids carrying either the Toxoplasma gondii dihydrofolate reductase gene with pyrimethamine or the human dhfr gene with WR99210 (Crabb and Cowman, 1996; Wu et al., 1996; Fidock and Wellems, 1997). Nevertheless, the introduction of DNA into the parasite genome is far from routine and the length of time needed for integration is a major obstacle to effective analysis. Hitherto all integration of DNA into the P. falciparum genome has been via single crossover recombination following extended periods of culture. A major impediment to the isolation of $P$. falciparum parasites with chromosomally integrated copies of the transformed plasmid is the persistence of circular forms of the plasmid. This has made it impossible to isolate parasites with gene disruptions that result in decreased growth rates.

The negative selection of cells expressing transformed 
genes has been developed in mammalian cells to increase the efficiency of gene targeting, and is proving useful for the selective killing of transformed cells in the field of cancer therapy (Mansour et al., 1988; Hoganson et al., 1996). Two commonly used genes include the thymidine kinase gene of Herpes simplex virus (Moolten, 1986) and the cytosine deaminase gene of Escherichia coli (Mullen et al., 1992). Negative selection relies on the expression of these genes in a cell that converts a normally innocuous metabolite into a toxic one. Viral thymidine kinase is a promiscuous enzyme that activates nucleoside analogues such as ganciclovir into toxic metabolites. Similarly, cytosine deaminase converts the pro-drug 5-fluorocytosine into 5-fluorouracil. The toxic metabolites produced by thymidine kinase and cytosine deaminase inhibit the de novo pyrimidine biosynthesis pathway and nucleic acid synthesis directly. The conversion of ganciclovir by thymidine kinase inhibits DNA synthesis and the enzyme thymidylate synthase, whereas the conversion of 5-fluorocytosine by cytosine deaminase inhibits RNA synthesis as well as the enzyme thymidylate synthase (Rogulski et al., 1997).

In pursuit of more efficient strategies for the genetic manipulation of the $P$. falciparum genome we have tested the utility of thymidine kinase and cytosine deaminase for the negative selection of $P$. falciparum. Following their stable introduction into $P$. falciparum an increase in sensitivity was observed to both ganciclovir and 5-fluorocytosine, respectively. Moreover, following selection with ganciclovir and WR99210 we were able to isolate $P$. falciparum transfectants with double crossover recombination events that had resulted in the genetic deletion of a portion of the Pfrh 3 gene. This locus was chosen because we have previously established it as a non-essential gene, which can be disrupted by the integration of plasmid DNA into this region through single crossover recombination (Taylor et al., 2001). The work described here describes the first targeted genetic deletion using negative selection and double crossover recombination in P. falciparum. These vectors will greatly facilitate the ability to analyse the $P$. falciparum genome and will be an important addition to the process of understanding the molecular basis of host-parasite interaction and identifying new vaccine candidates and drug targets.

\section{Methods and materials}

\subsection{Plasmid constructs}

The cytosine deaminase gene from E. coli was amplified using the primers 5'-GGACCGCTCGAGTTTTTATGTCGAATAACGCTTTACAAACAATT- ${ }^{\prime}$ and $5^{\prime}$-GGACCCTCGAGTCAACGTTTGTAGTCGATGGCTTCTGG- $3^{\prime}$. Following digestion of the PCR product with $X h o \mathrm{I}$ it was inserted into the $X h o I$ site of the plasmid transfection vector pHH1 (Reed et al., 2000), between the P. falciparum hsp86 promoter and the Plasmodium berghei dhfr-thymidylate synthase gene terminator, to give the construct $\mathrm{pHCD}$. Two DNA segments of approximately $1 \mathrm{~kb}$ from the Pfrh3 pseudogene (Taylor et al., 2001) (accession no. AF324831) were amplified from 3D7 genomic DNA and introduced into the flanking regions of the human $d h f r$ cassette to mediate the integration of the plasmid into the parasite genome (Fig. 1). The $5^{\prime}$ segment of Pfrh3 was amplified from genomic DNA of 3D7 parasites with the primers 5'-GGACCCCGCGGAAAACTTTCAGTTTTCAC- $3^{\prime}$ and $5^{\prime}$-GGACCGTTAACCTCCCAATATTCTCTTGTCC- $3^{\prime}$. This was introduced $5^{\prime}$ of the $h d h f r$ cassette
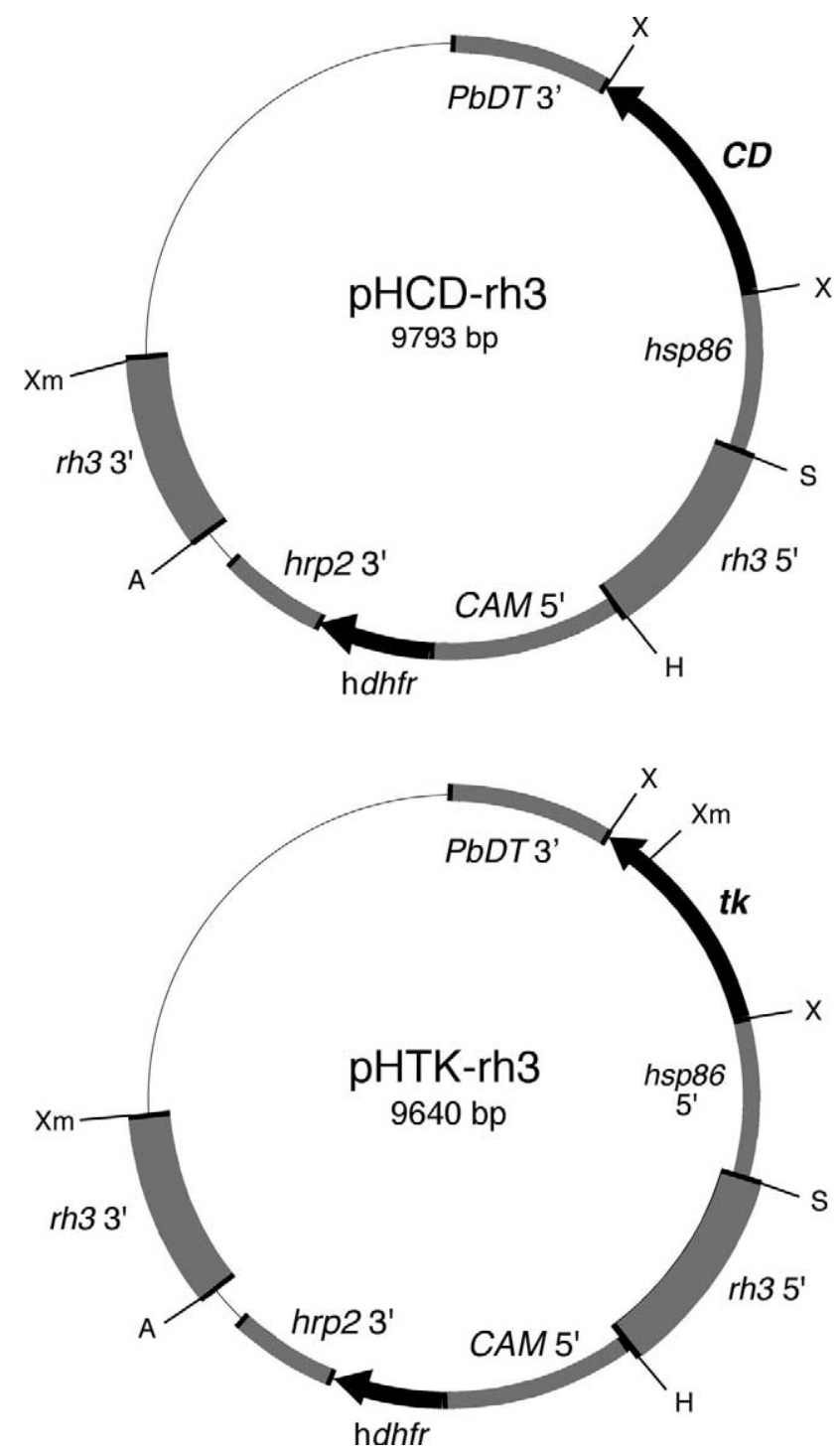

Fig. 1. Restriction maps of pHCD-rh3 and pHTK-rh3 transfection vectors for expression of thymidine kinase and cytosine deaminase in Plasmodium falciparum. $\mathrm{PbDT} 3^{\prime}$, P. berghei $3^{\prime}$ termination region; $\mathrm{CD}$, cytosine deaminase; hsp $865^{\prime}$, heat shock protein $865^{\prime}$ promoter; $r h 35^{\prime}, 5^{\prime}$ region of the Pfrh3 gene; CAM5 ${ }^{\prime}$, calmodulin promoter; h $d h f r$, human $d h f r$ gene; hrp2 $3^{\prime}$, histidine-rich protein $23^{\prime}$ termination region; rh3 $3^{\prime}, 3^{\prime}$ region of the Pfrh3 gene; tk, thymidine kinase gene. Restriction sites used for cloning in the Pfrh 3 gene flanks and the thymidine kinase and cytosine deaminase genes are shown. A, AvrII; H, HpaI; S, SacII; X, XhoI; Xm, XmaI. 
between the SacII and HpaI sites of pHCD. The $3^{\prime}$ segment of the Pfrh 3 gene was amplified with the primers $5^{\prime}$-GGACCACCGGTAGCCTAGGGACGGATTAGTTGAAAATAAATCC-3' and 5'-GGACCGGGCGCCCGGGTTTCCCATCAACTAAGG-3'. We were unable to introduce the $3^{\prime}$ fragment into the KasI site $3^{\prime}$ of the human dihydrofolate reductase gene cassette. Therefore using a linker, an XmaI site was introduced instead of the KasI site and the $3^{\prime}$ fragment cloned into this to give the plasmid pHCD-rh3 (Fig. 1). The thymidine kinase gene from Herpes simplex virus- 2 was amplified from the vector pTCTK (kindly provided by Dr Michael White, Montana State University) (Radke and White, 1999) using the primers 5'-GGACCGCTCGAGTTTTTATGGCTTCGTACCCCTGCCATCAAC- $3^{\prime}$ and 5'-GGACCGCTCGAGTCAGTTAGCCTCCCCCATCT$\mathrm{CCC}-3^{\prime}$ and cloned into the $\mathrm{pHCD}-\mathrm{rh} 3$ vector in place of the cytosine deaminase gene to give the plasmid pHTK-rh3 (Fig. 1). Both the thymidine kinase and cytosine deaminase genes were sequenced prior to transfection.

\subsection{Parasite growth and transfection}

Plasmodium falciparum asexual stages were maintained (Trager and Jensen, 1976) and sorbitol-synchronised (Lambros and Vanderberg, 1979) by standard procedures. 3D7 is a cloned line derived from NF54 and was obtained from Professor David Walliker at Edinburgh University. Predominantly ring-stage parasites were transfected with $100 \mu \mathrm{g}$ of purified (Qiagen) plasmid DNA as described previously (Fidock and Wellems, 1997). Positive selection was with $10 \mathrm{nM}$ WR99210, an antifolate drug which selects for the presence of the human $d h f r$ gene. Transformants were obtained between 15 and 23 days following transfection.

\subsection{Drugs and sensitivity assays}

WR99210 (kindly provided by Jacobus Pharmaceutical Co. Inc.) was dissolved in DMSO at a stock concentration of $20 \mathrm{mM}$ and stored at $-20^{\circ} \mathrm{C}$. Ganciclovir (provided by Cytovene) was dissolved in water at a concentration of 20 $\mathrm{mM}$ and stored at $-20^{\circ} \mathrm{C}$. Prior to use the stock tube was heated to $37^{\circ} \mathrm{C}$ for $5 \mathrm{~min}$ to dissolve precipitated drug. 5fluorocytosine (Sigma) was made fresh for every assay. The drug was dissolved at $1-2 \mathrm{mg} / \mathrm{ml}$ directly into hypoxanthine-free complete medium, heated to $50^{\circ} \mathrm{C}$ for $10 \mathrm{~min}$ to dissolve the drug, and then filter-sterilised using a $20-\mu \mathrm{m}$ filter prior to use. The drug sensitivity assays were carried out as described previously with some modifications (Thaithong and Beale, 1981). Briefly, synchronised ringstage parasites were cultured at a starting parasitaemia of 1 and $2.5 \%$ haematocrit for $72 \mathrm{~h}$. At $48 \mathrm{~h}$ fresh medium containing $0.2 \mu \mathrm{Ci}$ tritiated-hypoxanthine was added to each well. The cells were harvested at $72 \mathrm{~h}$ and c.p.m. were determined on a TopCount 1 scintillation machine. Percentage inhibition was determined as the c.p.m. from each well relative to the average of six control wells without drug. These assays were carried out three times in duplicate.

The presence of a 'bystander effect' was tested by mixing wild-type 3D7 parasites at different proportions (1:0, 3:1, $1: 11: 3,0: 1)$ with parasites expressing either thymidine kinase or cytosine deaminase at a final parasitaemia of $1 \%$ and haematocrit of $2.5 \%$. Minimum inhibitory concentrations of ganciclovir and 5-fluorocytosine were added to each well. Percentage inhibition relative to wells without drug was calculated as above.

\subsection{DNA, chromosomes and protein analysis of parasites}

Genomic DNA and intact chromosomes were prepared from trophozoites as described (Triglia and Cowman, 1994). Pulsed-field gel electrophoresis to separate chromosomes was performed as previously described (Rubio et al., 1996). Southern blotting was carried out using standard procedures. Either unsynchronised or synchronised 3D7 parasites were cultured for approximately $36 \mathrm{~h}$ until they were late schizonts. Protein preparations of these parasites were analysed by SDS-PAGE on $6 \%$ polyacrylamide gels and transferred to nitrocellulose as described (Triglia and Cowman, 1994). Primary rabbit antibodies were initially bound to the transferred proteins, and bound antibody was detected with horse-radish peroxidase coupled sheep antirabbit IgG (Silenus Laboratories) and developed by ECL, a luminescence-based method (Amersham International). Rabbit anti- $H S V-1$ thymidine kinase antibodies were kindly provided by Professor W.C. Summers (Yale University). Anti-E. coli cytosine deaminase antibodies were kindly provided by Dr C. Richards (Glaxo-Wellcome).

\section{Results}

\subsection{Transformation of P. falciparum blood stages with episomal plasmids encoding the Herpes simplex thymidine kinase or the E. coli cytosine deaminase gene}

In order to obtain genetic deletions in P. falciparum via double crossover recombination a plasmid containing a drug resistance marker for positive selection flanked by two target sequences at the $5^{\prime}$ and $3^{\prime}$ ends is required. Additionally, it would be essential to include a second gene for negative selection outside of this targeting cassette for homologous recombination to circumvent the maintenance of episomal plasmids. Therefore we have constructed a transfection plasmid containing the $h d h f r$ gene under the control of the P. falciparum calmodulin (Pfcam) promoter that could be positively selected with the drug WR99210. Two fragments of the Pfrh 3 gene $(\sim 1.0 \mathrm{~kb}$ each) (Taylor et al., 2001) were cloned at the 5' and $3^{\prime}$ ends of the hdhfr gene so that two target sequences for homologous recombination by double crossover were present in the plasmid. In order to test whether thymidine kinase or cytosine deaminase could be expressed in P.falciparum and used to negatively select 
maintenance of the plasmid backbone we inserted the corresponding genes between the Pfhsp 86 promoter and the $3^{\prime}$ terminator region of the P.berghei dhfr gene to derive the vectors pHCD-rh3 and pHTK-rh3 (Fig. 1).

Both pHCD-rh3 and pHTK-rh3 were transfected separately into the 3D7 cloned line and parasites containing those plasmids were positively selected with WR99210. In order to determine if the thymidine kinase and cytosine deaminase proteins were expressed in 3D7/pHCD-rh3 and 3D7/pHTK-rh3, respectively, Western blots were performed using mixed stage parasites and anti-cytosine deaminase and anti-thymidine kinase antibodies (Fig. 2B,D). Expression of cytosine deaminase and thymidine kinase was detected in 3D7/pHCD-rh3 and 3D7/pHTKrh3, respectively, whereas no detectable protein was observed in the parental 3D7 parasite line. These results confirm that we are able to express both thymidine kinase and cytosine deaminase in $P$. falciparum under the control of the Pfhsp 86 promoter.

\subsection{Plasmodium falciparum parasites expressing cytosine} deaminase or thymidine kinase are sensitive to 5 fluorocytosine and ganciclovir respectively

To test if the $P$. falciparum transfected parasites expressing cytosine deaminase or thymidine kinase had an altered sensitivity to the pro-drugs 5-fluorocytosine or ganciclovir, we performed drug sensitivity assays on the 3D7/pHCD-rh3 and 3D7/pHTK-rh3 transformants for comparison with parental 3D7 (Fig. 2A,C). The 3D7 parental line was insen-
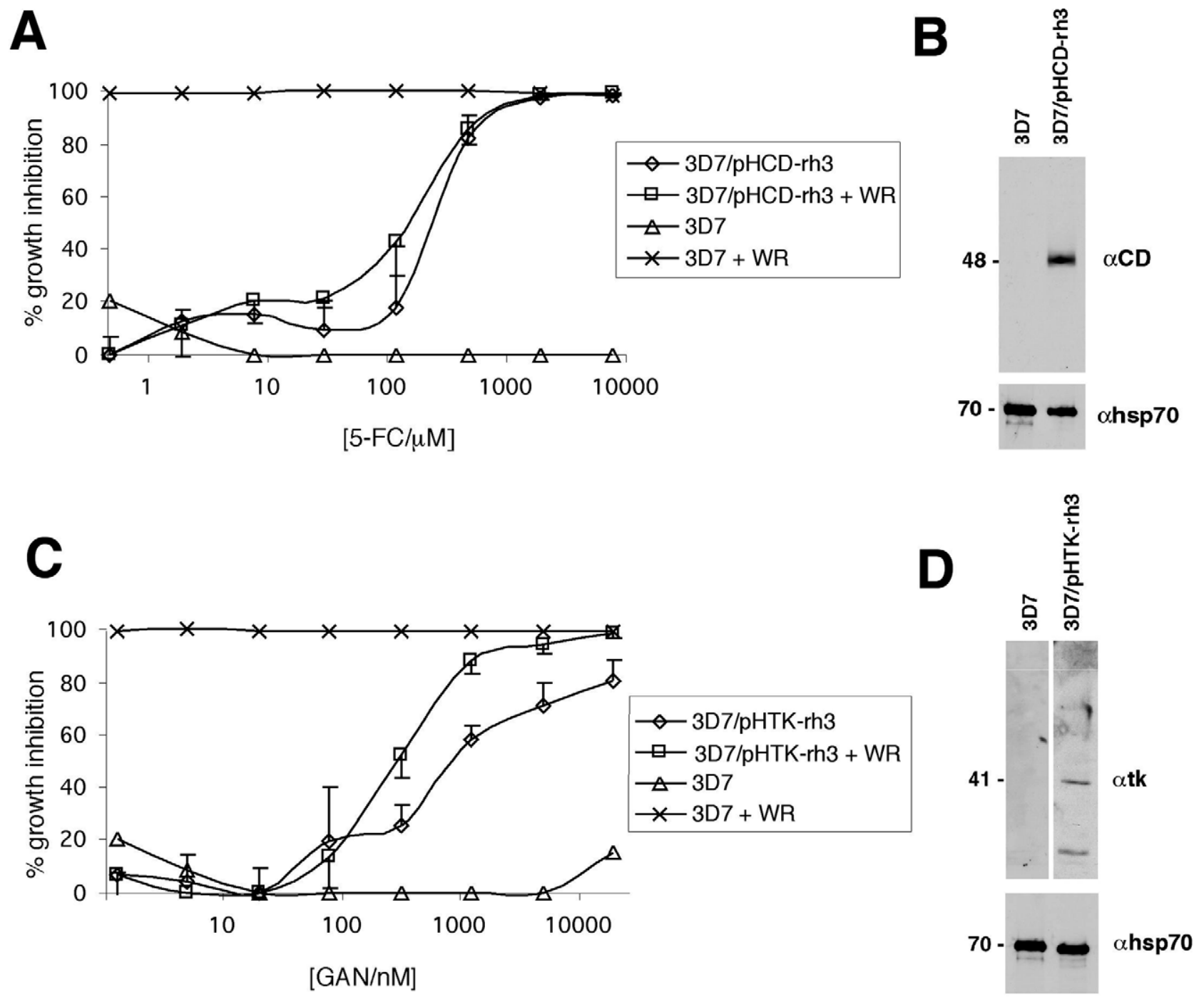

Fig. 2. Drug sensitivity and expression of thymidine kinase and cytosine deaminase in Plasmodium falciparum. (A) Inhibition of 3D7 and 3D7/pHCD-rh3 with 5-fluorocytosine (5-FC) and/or WR99210. 3D7 parasites transformed with the pHTK-rh3 and pHCD-rh3 plasmids were obtained by WR99210 selection. (B) Expression of cytosine deaminase in 3D7-pHCD-rh3 compared to the parental line 3D7. (C) Inhibition of 3D7 and 3D7/pHTK-rh3 with ganciclovir (GAN) and/or WR99210. (D) Expression of thymidine kinase in 3D7-pHTK-rh3 compared to the parental line 3D7. Protein preparations obtained from unsynchronised cultures of each transformant were separated by SDS-PAGE, electro-blotted, then probed with anti-thymidine kinase and anti-cytosine deaminase antibodies. Protein sizes are shown in kDa. 
sitive to the levels of 5-fluorocytosine tested, but as expected highly sensitive to WR99210 as it did not contain the $h d h f r$ gene. In comparison the transfected line 3D7/ pHCD-rh3, which expressed cytosine deaminase was sensitive to 5-fluorocytosine in a dose-dependent fashion with an $\mathrm{IC}_{50}$ of $233 \mu \mathrm{M}$. The $\mathrm{IC}_{50}$ of 5-fluorocytosine decreased to $124 \mu \mathrm{M}$ in the presence of WR99210.

The 3D7/pHTK-rh3 transfected parasite line was found to be sensitive to ganciclovir with an $\mathrm{IC}_{50}$ of $1.14 \mu \mathrm{M}$ in comparison to the parental line 3D7 which was insensitive at the concentrations tested (Fig. 2C). Interestingly, the $\mathrm{IC}_{50}$ of 3D7/pHTK-rh3 for 5-fluorocytosine decreased significantly to $0.3 \mu \mathrm{M}$ in the presence of WR99210, suggesting a strong degree of synergism for the drug combination. These results confirm that cytosine deaminase and thymidine kinase can both be used for negative selection in $P$. falciparum with 5-fluorocytosine and ganciclovir, respectively. As additional controls 3D7/pHCD-rh3 was found to be totally insensitive to ganciclovir, while conversely 3D7/ pHTK-rh3 was insensitive to 5-fluorocytosine, indicating that the negative selection was specific to each negative selectable marker and was not mediated by the $h d h f r$ gene.

The drug sensitivity assays were repeated for both 3D7/ pHCD-rh3 and 3D7/pHTK-rh3 and the parasites were followed microscopically. This confirmed that the nature of the inhibition for both 5-fluorocytosine and ganciclovir was cytostatic with a growth arrest during schizogony. Removal of 5-fluorocytosine or ganciclovir resulted in ablation of the growth arrest confirming the cytostatic nature of the inhibition.

\subsection{The 3D7/pHCD-rh3 and 3D7/pHTK-rh3 transfectants exhibit a 'bystander effect'}

Previously it has been shown that when thymidine kinase or cytosine deaminase are expressed in a fraction of the cells in an in vitro mammalian cell culture, that on addition of either 5-fluorocytosine or ganciclovir some of the cells not expressing the negative selectable markers were killed alongside those expressing them. This 'bystander effect' is thought to be due to the diffusion of toxic metabolites produced in the transformed cells to neighbouring cells (Freeman et al., 1993; Hoganson et al., 1996). It was important to determine if a 'bystander effect' can occur in $P$. falciparum parasites expressing thymidine kinase or cytosine deaminase as it will be essential to take this into account when negatively selecting for transfectants on 5fluorocytosine or ganciclovir. 3D7 parasites were genotypically negative for thymidine kinase or cytosine deaminase were mixed at different ratios with genotypically positive 3D7/pHCD-rh3 or 3D7/pHTK-rh3 transformants. These cells were grown in minimum inhibitory concentrations of 5-fluorouracil or ganciclovir. Both thymidine kinase and cytosine deaminase expressing parasites mediate a 'bystander' effect, with the genotypically negative 3D7 cells being inhibited to a much greater degree than expected in the absence of a bystander effect (Fig. 3).

\subsection{Negative and positive selection for disruption of the P. falciparum Pfrh3 gene}

We selected $P$. falciparum transfectants containing episomal copies of the pHCD-rh3 or pHTK-rh3 plasmids with
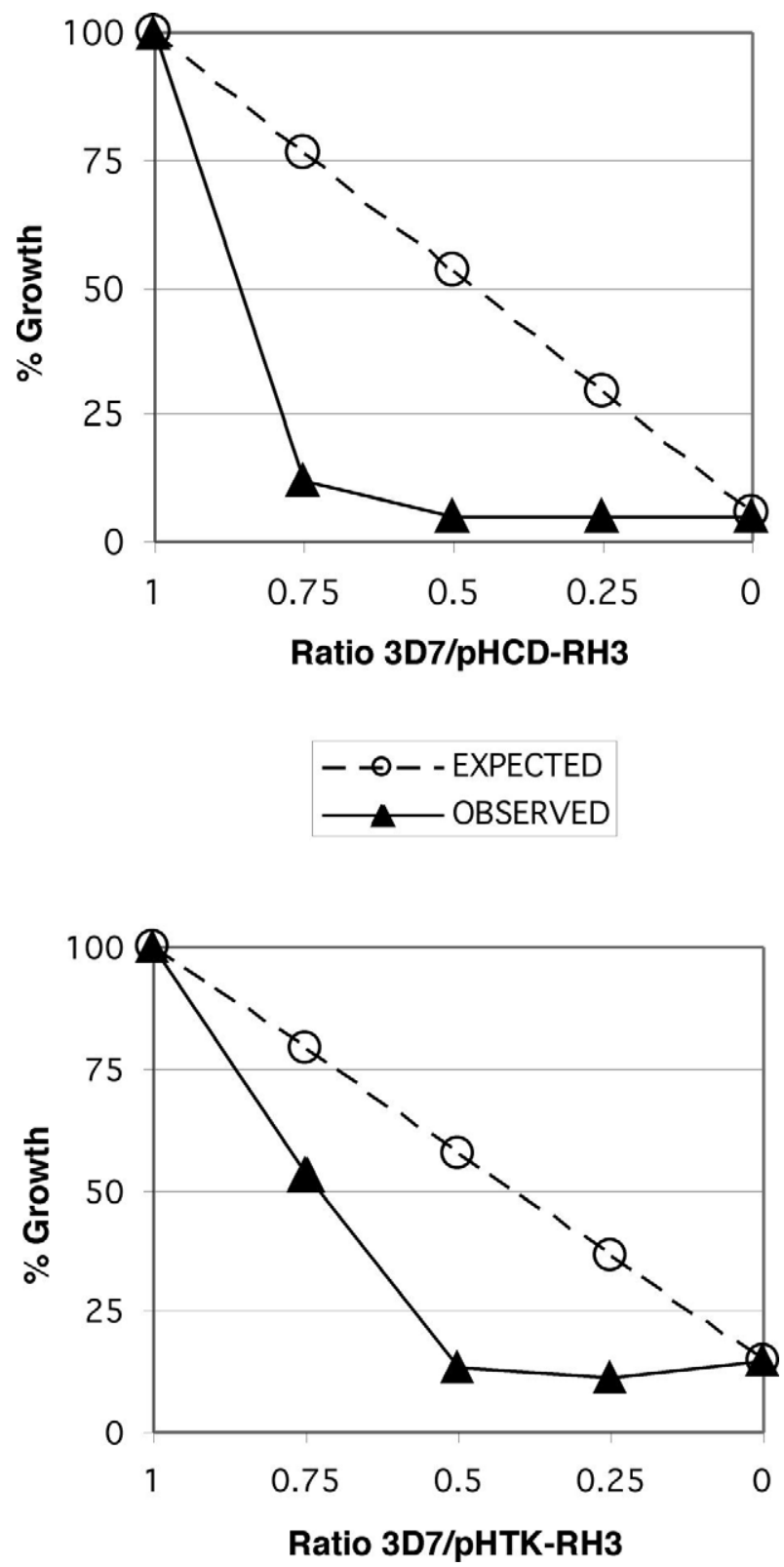

Fig. 3. The 'bystander effect' seen for 3D7/pHCD-rh3 and 3D7/pHTK-rh3. 'Observed': actual \% parasite growth compared with untreated controls when 3D7 parasites were mixed in the ratios indicated with either pHCD-rh3 parasites in MIC concentrations of 5-fluorocytosine or with pHTK-rh3 parasites in MIC concentrations of ganciclovir. 'Expected': \% growth of the parasites expected in the absence of a bystander effect when mixed in the ratios indicated, derived from the \% growth of 3D7 and pHCD-rh3 or pHTK-rh3 parasites when grown in MIC concentrations of either 5-fluorocytosine or ganciclovir separately. 
either 5-fluorocytosine or ganciclovir and 5 nM WR99210, respectively, to determine if selection of genetic deletion of a portion of the Pfrh3 gene by double crossover recombination could be achieved. Several protocols were used which take into account the 'bystander effect' and the likely possibility that the double crossover recombination is a very low frequency event. These included selection with minimum inhibitory concentration (MIC) or $\mathrm{IC}_{50}$ values of either 5-

\section{A}
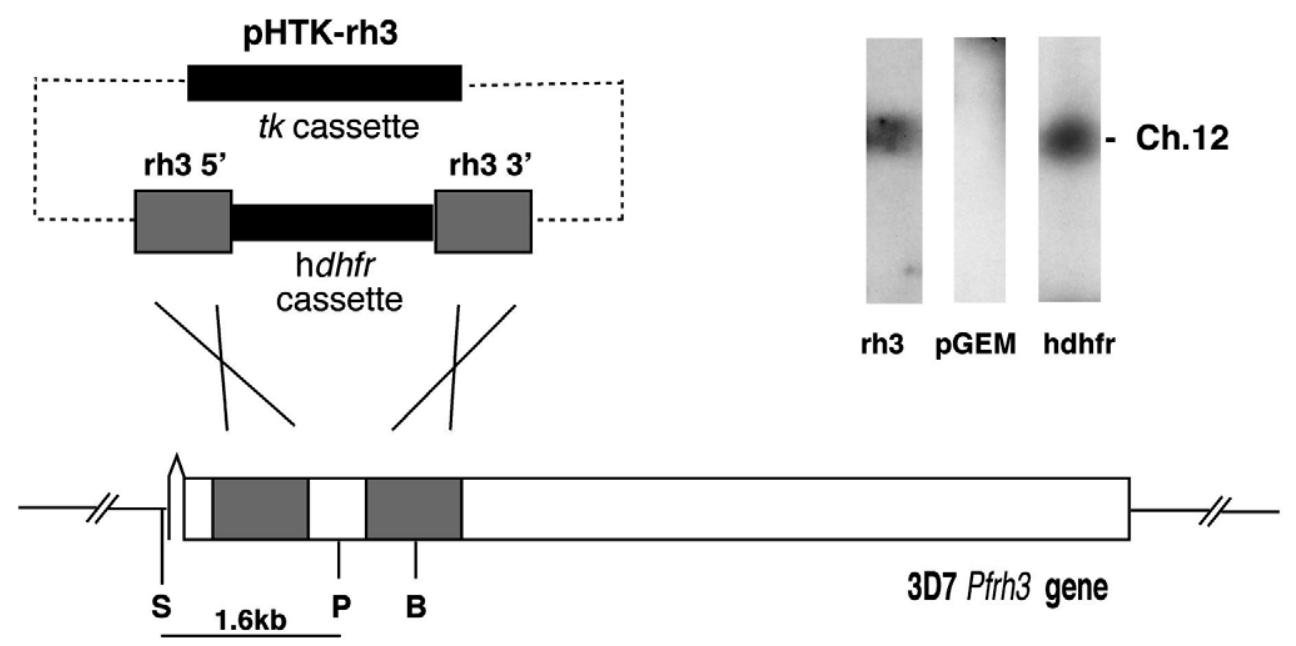

$2.5 \mathrm{~kb}$

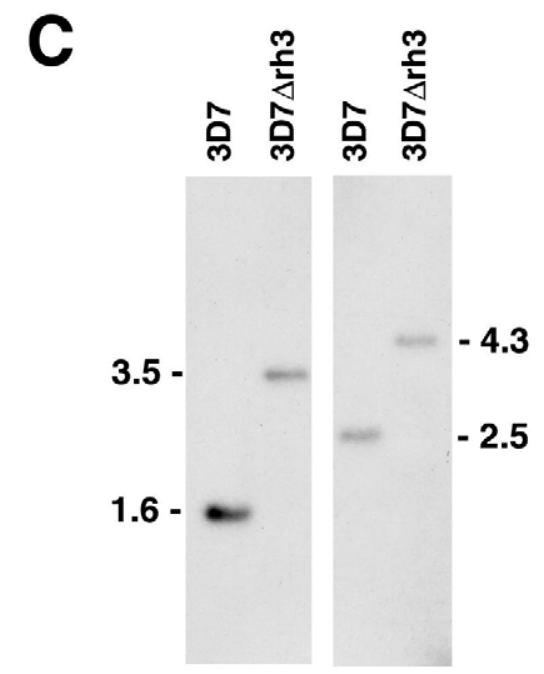

B 3D7هrh3

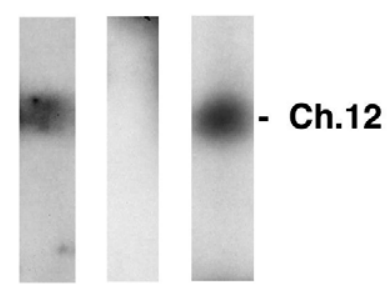

rh3 pGEM hdhfr
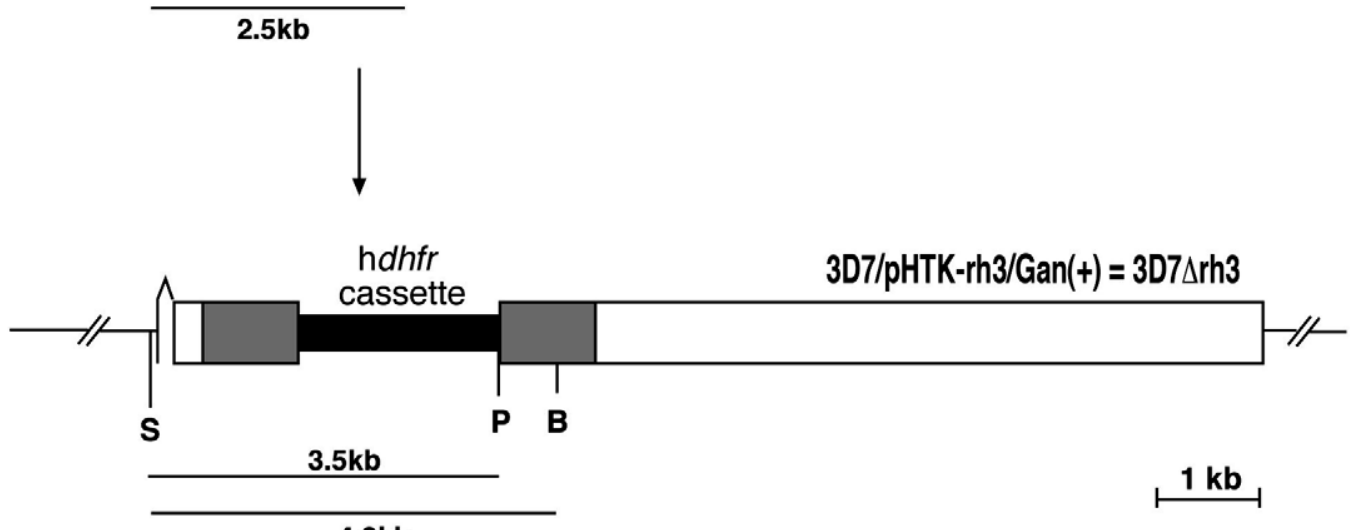

$4.3 \mathrm{~kb}$

\section{3}

2.5
D

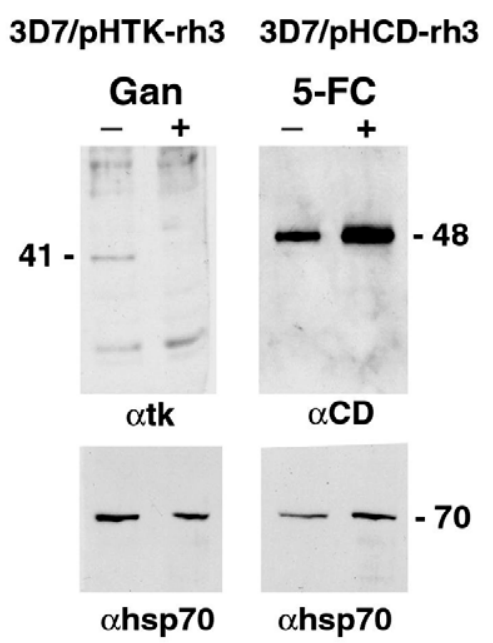


fluorocytosine or ganciclovir at starting parasitaemias of 0.5 and $4 \%$ haematocrit. Parasites expressing cytosine deaminase were killed when selected continuously with MIC or $\mathrm{IC}_{50}$ concentrations of 5-fluorocytosine with WR99210, with no parasites visible up to 6 weeks following the initiation of selection. However, parasites were obtained using an intermittent drug selection. These parasites were insensitive to 5-fluorocytosine but on further analysis were found to contain the episomal plasmid and still expressed cytosine deaminase (Fig. 4D). We were unable to successfully select double recombination events into the Pfrh 3 gene using cytosine deaminase as the negative selectable marker.

Following selection of 3D7/TK-rh3, parasites expressing thymidine kinase with MIC values of ganciclovir initially appeared to die as expected but reappeared after 6 days of subsequent culture. Similarly, when selected on $\mathrm{IC}_{50}$ values of ganciclovir a $50 \%$ reduction in growth compared with a control was observed in three subsequent cycles until this was then ablated. These parasites (3D7Drh3) were subsequently analysed and found to be insensitive to ganciclovir and resistant to WR99210 as would be expected for transfectants that had inserted the hdhfr gene but deleted the thymidine kinase gene of the plasmid via a double crossover recombination mechanism.

In order to test if the hdhfr gene had been inserted into the Pfrh3 gene on chromosome 12 by double crossover recombination we analysed chromosomes by pulsed field gel electrophoresis (PFGE) (Fig. 4B) and genomic DNA using Southern blots (Fig. 4C). Hybridisation of chromosomes from the $3 \mathrm{D} 7 \Delta \mathrm{rh} 3$ parasites with a Pfrh3 probe confirmed the presence of this gene on chromosome 12 . No hybridisation was detected to chromosome 12 with the pGEM plasmid backbone, indicating that this region had not integrated. However, the $h d h f r$ probe did hybridise to chromosome 12, confirming that this region of the plasmid had integrated, consistent with insertion into the Pfrh3 gene by double crossover recombination (Fig. 4A). Southern analysis of genomic DNA from Pfrh3 with a number of restriction enzymes confirmed that a portion of the Pfrh3 gene had been deleted via a double crossover recombination event, resulting in deletion of the plasmid backbone and the thymi- dine kinase gene (Fig. 4C). This is consistent with 3D7 $\Delta$ rh3 parasites being resistant to ganciclovir and WR99210 and the lack of expression of thymidine kinase in these parasites compared with other parasites not exposed to ganciclovir (Fig. 4D). In the course of the study we discovered that the 3D7/pHTK-rh3 line prior to negative selection contained a mixture of episomal with some single crossover recombinant parasites. This was not surprising as we have previously observed single crossover integration into the Pfrh3 locus (Taylor et al., 2001). The negative selection with ganciclovir therefore selects against single crossover recombinants of the thymidine kinase gene as well as episomal forms. Subsequently using this system we have made several gene disruptions in P. falciparum by double crossover recombination in parasites that had only episomal plasmid and no detectable single recombination events (data not shown).

\section{Discussion}

In this study we have expressed both the Herpes simplex thymidine kinase and the E. coli cytosine deaminase genes in P. falciparum asexual blood stages and demonstrated that they can act as negative selectable markers. Using this strategy with thymidine kinase we have been able to derive a genetic deletion that has inserted the drug selection cassette via double crossover recombination. This is the first time this has been achieved in $P$. falciparum and the use of this vector system will allow selection of gene knockouts in $P$. falciparum with deleterious phenotypes. The persistence of transfection plasmids in P. falciparum has been a major impediment to functional analysis, which should be circumvented by the use of the approach described here. Additionally, the frequency of double crossover recombination is high enough in blood-stage parasites to allow for their selection.

Expression of thymidine kinase or cytosine deaminase confers on $P$. falciparum sensitivity to ganciclovir and 5fluorocytosine, respectively, and this was the key to providing a system that would negatively select against the persis-

Fig. 4. The Pfrh3 gene is disrupted by a double-crossover event. (A) Schematic representation of the Pfrh3 locus in the $3 \mathrm{D} 7 \Delta \mathrm{rh} 3$ parasites. The plasmid construct, pHTK-rh3, contains two portions of the Pfrh3 gene (shaded portions) which were cloned in the same direction flanking the hdhfr cassette. The pGEM vector backbone is shown as the dashed line. The crosses indicate the two regions where homologous integration has occurred. The restriction sites shown are $S p h \mathrm{I}(\mathrm{S}), B g l \mathrm{II}(\mathrm{B})$ and $P v u \mathrm{II}(\mathrm{P})$. (B) The three largest chromosomes of the $3 \mathrm{D} 7 \Delta \mathrm{rh} 3$ parasites were resolved by pulsed-field gel electrophoresis in a $1 \times \mathrm{TAE}$, $1 \%$ FastLane (Seakem) agarose gel for $96 \mathrm{~h}$ at $3 \mathrm{~V} / \mathrm{cm}$ with a switch time of 360-800 s and a linear ramp using a contour clamped homogeneous electric field (CHEF) apparatus (Rubio et al., 1995). Chromosomal DNAs were blotted to hybond N then probed with DNA fragments corresponding to Pfrh3, pGEM and human $d h f r$. (C) Southern analysis of untransfected 3D7 and Pfrh3-disrupted 3D7 parasites (3D7Drh3).The left panel shows genomic DNAs digested with SphI and $P v u I I$, while the right panel shows a SphI/BglII double digest. The Southern blot was probed with a 303 bp Pfrh3 fragment within the Pfrh3 $5^{\prime}$ flank. The fragment was amplified with the primers $5^{\prime}$-AAAACTTTCAGTTTTCAC-3 ${ }^{\prime}$ and $5^{\prime}$-TGGGGACCCGTTACCTTTA- ${ }^{\prime}$. Sizes are shown in kb. (D) Expression of thymidine kinase and cytosine deaminase in ganciclovir- and 5-fluorocytosine-treated 3D7/pHTK-rh3 and 3D7/pHCD-rh3 parasites. Both the pHTK-rh3and pHCD-rh3-transfected parasites were maintained on WR99210. The 3D7/pHTK-rh3 parasites were either not treated or treated with $4 \mu \mathrm{M}$ ganciclovir for 6 days until parasites re-appeared. Similarly, the pHCD-rh3 parasites were either not treated or treated intermittently with $300 \mu \mathrm{M} 5$-FC for 4 days followed by drug-free recovery periods, until resistant parasites were obtained. Protein samples taken from parasites synchronised to the late schizont stage with or without ganciclovir or 5-fluorocytosine treatment were separated by SDS-PAGE, electro-blotted and probed with anti-thymidine kinase or anti-cytosine deaminase antibodies as appropriate. In addition, the same blots were probed with anti-hsp70 antibodies as a protein loading control. Sizes shown are in kDa. 
tence of episomal plasmids in transfectants. We were unable to obtain $P$. falciparum transfectants that had inserted the plasmids via double crossover recombination using cytosine deaminase and instead obtained resistance to 5-fluorocytosine. Sequencing of the cytosine deaminase gene recovered from plasmids rescued from these parasites did not identify any mutations and it is possible that alterations in other proteins such as transporters may be responsible for this resistance. It may be possible to use cytosine deaminase for the selection of double crossover recombinants, particularly by plating the cells for negative selection at a lower density as is required for the generation of embryonic stem cell deletions and other strategies to minimise the emergence of natural mutants within the population.

The demonstration that thymidine kinase and cytosine deaminase expression in $P$. falciparum show a marked 'bystander effect' would decrease the possibility of selecting low frequency double crossover recombinants due to the indiscriminate killing of these cells (Freeman et al., 1993; Hirschowitz et al., 1995). The expression of a 'bystander effect' in P. falciparum is surprising since the same effect in mammalian cells has been attributed to the diffusion of toxic metabolites via gap junctions (Freeman et al., 1993). In the case of $P$. falciparum it is unlikely that the metabolites can be transported out of the multiple membranes and instead, we believe that the toxic metabolites are released into the intercellular milieu during the disintegration of the cell and are then taken up by rapidly growing parasite cells.

Interestingly, a synergistic effect was observed when both the positive selection drug WR99210 and the negative selection drug ganciclovir were used together against $P$. falciparum cells expressing thymidine kinase. This may be due to the proximity of the enzymes dihydrofolate reductase and thymidylate synthase in the enzyme pathways involved in folate metabolism. In fact in $P$. falciparum the two enzyme functions dihydrofolate reductase and TS reside on the same polypeptide (Bzik et al., 1987). WR99210 inhibits dihydrofolate reductase (Fidock and Wellems, 1997) while the toxic metabolites of ganciclovir inhibit thymidylate synthase.

We have been unable to derive transfectants with plasmid insertion via double crossover recombination directly from transfecting linearised plasmid DNA despite numerous attempts using different strategies. This may be due to the low efficiency of initial transfection in $P$. falciparum and the decreased frequency expected for double crossover recombination compared to that seen for single crossover recombination (Crabb et al., 1997b). Importantly, we cannot rule out the possibility that double crossover recombination occurs by a two-step process through a single crossover recombination intermediate. Practically this may be of little consequence provided there is a small population of parasites containing integration via single recombination combined with the power of the thymidine kinase -mediated negative selection. Furthermore, single crossover recombinants may be generated as a first step by non-deleterious $3^{\prime}$ replacements (Triglia et al., 1997) which can then be followed by a subsequent negative selection step for the double crossover recombinant. This approach has the advantage of verifying whether the locus of interest can be effectively targeted.

The Pfrh 3 gene has been shown previously to be a transcribed pseudogene (Taylor et al., 2001), so it is not surprising that we were able to select for parasites with a portion of this gene deleted. However, this does provide an example of the utility of this negative selection strategy. Not only does the use of negative selection increase the possibility of obtaining the desired gene disruption events but also drastically reduces the time required to select the parasites with the integration events. This is because it becomes almost unnecessary to grow the transfected parasites on cycles on and off drug selection to 'cure' the parasites of the episomal plasmid (Crabb and Cowman, 1996; Wu et al., 1996.

The ability to use negative selection with thymidine kinase is an important advance in our ability to construct 'loss of function' mutations as it will allow the selection of gene disruption events that are deleterious to the growth of the parasite. This is important as these are the mutants that are most likely to provide information on the function of the protein encoded by these genes. Additionally, the demonstration that both thymidine kinase and cytosine deaminase can act as negative selectable markers has opened up the interesting possibility of using these genes to select regions of the genome that are silenced or activated. This will be an important approach to analyse the mechanism of silencing for $P$. falciparum gene families such as var and rifins. The development of this vector system will allow more efficient exploitation of the information provided by the genome of $P$. falciparum and opens the possibility for a concerted program of gene knockouts of the whole genome of this important pathogen. This will not only provide the tools to more fully understand the biology of this parasite but will also aid in the identification of new vaccine and antimalarial drug targets.

\section{Acknowledgements}

We thank Brendan Crabb for a critical review of the manuscript. This work was supported by the National Health and Medical Research Council of Australia (NH\&MRC). M.T.D. is a recipient of a Wellcome Trust Advanced Training Fellowship (Tropical Medicine) and A.F.C. is an International Research Scholar from the Howard Hughes Medical Institute. We would like to thank the Red Cross Blood Service (Melbourne, Australia) for the supply of red blood cells and serum.

\section{References}

Bowman, S., Lawson, D., Basham, D., Brown, D., Chillingworth, T., Churcher, C.M., Craig, A., Davies, R.M., Devlin, K., Feltwell, T., Gentles, S., Gwilliam, R., Hamlin, N., Harris, D., Holroyd, S., Hornsby, 
T., Horrocks, P., Jagels, K., Jassal, B., Kyes, S., McLean, J., Moule, S., Mungall, K., Murphy, L., Oliver, K., Quail, M.A., Rajandream, M.-A., Rutter, S., Skelton, J., Squares, R., Squares, S., Sulston, J.E., Whitehead, S., Woodward, J.R., Newbold, C., Barrell, B.G., 1999. The complete nucleotide sequence of chromosome 3 of Plasmodium falciparum. Nature, 532-8.

Bzik, D.J., Li, W.-B., Horii, T., Inselburg, J., 1987. Molecular cloning and sequence analysis of the Plasmodium falciparum dihydrofolate reductase-thymidylate synthase gene. Proc. Natl. Acad. Sci. USA 84, 8360-4.

Crabb, B.S., Cowman, A.F., 1996. Characterization of promoters and stable transfection by homologous and nonhomologous recombination in Plasmodium falciparum. Proc. Natl. Acad. Sci. USA 93, 7289-94.

Crabb, B.S., Cooke, B.M., Reeder, J.C., Waller, R.F., Caruana, S.R., Davern, K.M., Wickham, M.E., Brown, G.V., Coppel, R.L., Cowman, A.F., 1997a. Targeted gene disruption shows that knobs enable malariainfected red cells to cytoadhere under physiological shear stress. Cell 89, 287-96.

Crabb, B.S., Triglia, T., Waterkeyn, J.G., Cowman, A.F., 1997b. Stable transgene expression in Plasmodium falciparum. Mol. Biochem. Parasitol. 90, 131-44.

Fidock, D.A., Wellems, T.E., 1997. Transformation with human dihydrofolate reductase renders malaria parasites insensitive to WR99210 but does not affect the intrinsic activity of proguanil. Proc. Natl. Acad. Sci. USA 94, 10931-6.

Freeman, S.M., Abboud, C.N., Whartenby, K.A., Packman, C.H., Koeplin, D.S., Moolten, F.L., Abraham, G.N., 1993. The 'bystander effect': tumor regression when a fraction of the tumor mass is genetically modified. Cancer Res. 53, 5274-83.

Gardner, M.J., Tettelin, H., Carucci, D.J., Cummings, L.M., Aravind, L., Koonin, E.V., Shallom, S., Mason, T., Yu, K., Fujii, C., Pederson, J., Shen, K., Jing, J., Aston, C., Lai, Z., Schwartz, D.C., Pertea, M., Salzberg, S., Zhou, L., Sutton, G.G., Clayton, R., White, O., Smith, H.O., Fraser, C.M., Adams, M.D., Venter, J.C., Hoffman, S.L., 1998. Chromosome 2 sequence of the human malaria parasite Plasmodium falciparum. Science 282, 1126-32.

Hirschowitz, E.A., Ohwada, A., Pascal, W.R., Russi, T.J., Crystal, R.G., 1995. In vivo adenovirus-mediated gene transfer of the Escherichia coli cytosine deaminase gene to human colon carcinoma-derived tumors induces chemosensitivity to 5-fluorocytosine. Hum. Gene Ther. 6, 1055-63.

Hoganson, D.K., Batra, R.K., Olsen, J.C., Boucher, R.C., 1996. Comparison of the effects of three different toxin genes and their levels of expression on cell growth and bystander effect in lung adenocarcinoma. Cancer Res. 56, 1315-23.

Lambros, C., Vanderberg, J.P., 1979. Synchronization of Plasmodium falciparum erythrocytic stages in culture. . J. Parasitol. 65, 418-20.

Mansour, S.L., Thomas, K.R., Capecchi, M.R., 1988. Disruption of the proto-oncogene int-2 in mouse embryo-derived stem cells: a general strategy for targeting mutations to non-selectable genes. Nature 336, $348-52$.

Moolten, F.L., 1986. Tumor chemosensitivity conferred by inserted herpes thymidine kinase genes: paradigm for a prospective cancer control strategy. Cancer Res. 46, 5276-81.

Mullen, C.A., Kilstrup, M., Blaese, R.M., 1992. Transfer of the bacterial gene for cytosine deaminase to mammalian cells confers lethal sensitivity to 5-fluorocytosine: a negative selection system. Proc. Natl. Acad. Sci. USA 89, 33-37.

Radke, J.R., White, M., 1999. Expression of herpes simplex virus thymidine kinase in Toxoplasma gondii attenuates tachyzoite virulence in mice. Infect. Immun. 67, 5292-7.

Reed, M.B., Saliba, K.J., Caruana, S.R., Kirk, K., Cowman, A.F., 2000. Pgh1 modulates sensitivity and resistance to multiple antimalarials in Plasmodium falciparum. Nature 403, 906-9.

Rogulski, K.R., Kim, J.H., Kim, S.H., Freytag, S.O., 1997. Glioma cells transduced with an Escherichia coli CD/HSV-1 TK fusion gene exhibit enhanced metabolic suicide and radiosensitivity. Hum. Gene Ther. 8, $73-85$.

Rubio, J.P., Triglia, T., Kemp, D.J., de Bruin, D., Ravetch, J.V., Cowman, A.F., 1995. A YAC contig map of Plasmodium falciparum chromosome 4: characterization of a DNA amplification between two recently separated isolates. Genomics 26, 192-8.

Rubio, J.P., Thompson, J.K., Cowman, A.F., 1996. The var genes of Plasmodium falciparum are located in the subtelomeric region of most chromosomes. EMBO J. 15, 4069-77.

Taylor, H.M., Triglia, T., Thompson, J., Sajid, M., Fowler, R., Wickham, M.E., Cowman, A.F., Holder, A.A., 2001. Plasmodium falciparum homologue of the genes for Plasmodium vivax and Plasmodium yoelii adhesive proteins, which is transcribed but not translated. Infect. Immun. 69, 3635-45.

Thaithong, S., Beale, G.H., 1981. Resistance of ten Thai isolates of Plasmodium falciparum to chloroquine and pyrimethamine by in vitro tests. Trans. R. Soc. Trop. Med. Hyg. 75, 271-3.

Trager, W., Jensen, J.B., 1976. Human malaria parasites in continuous culture. Science 193, 673-5.

Triglia, T., Cowman, A.F., 1994. Primary structure and expression of the dihydropteroate synthetase gene of Plasmodium falciparum. Proc. Natl. Acad. Sci. USA 91, 7149-53.

Triglia, T., Menting, J.G.T., Wilson, C., Cowman, A.F., 1997. Mutations of dihydropteroate synthase are responsible for sulfone and sulfonamide resistance in Plasmodium falciparum. Proc. Natl. Acad. Sci. USA 94, 13944-9.

Triglia, T., Wang, P., Sims, P.F.G., Hyde, J.E., Cowman, A.F., 1998. Allelic exchange at the endogenous genomic locus in Plasmodium falciparum proves the role of dihydropteroate synthase in sulfadoxineresistant malaria. EMBO J. 17, 3807-15.

Waller, R.F., Reed, M.B., Cowman, A.F., McFadden, G.I., 2000. Protein trafficking to the plastid of Plasmodium falciparum is via the secretory pathway. EMBO J. 19, 1794-802.

Wu, Y., Kirkman, L.A., Wellems, T.E., 1996. Transformation of Plasmodium falciparum malaria parasites by homologous integration of plasmids that confer resistance to pyrimethamine. Proc. Natl. Acad. Sci. USA $93,1130-4$. 